\title{
OVERCOMING THE SNR-WALL BY AUTOCORRELATION BASED SENSING OF OFDM SIGNAL
}

\author{
Sukanta Mondal ${ }^{1}$, Sandip Karar ${ }^{2}$ Partha Banerjee ${ }^{3}$, Arindam Biswas ${ }^{4 *}$, Anup \\ Kumar Bhattacharya ${ }^{4}$ \\ ${ }^{1,4}$ Dept of ECE, NIT, Durgapur, India \\ ${ }^{2}$ Institute of Radio Physics and Electronics, University of Calcutta, India \\ ${ }^{3}$ Academy of Technology, Hooghly, West Bengal, India \\ ${ }^{4}$ School of Mines and Metallurgy, Kazi Nazrul University, Asansol, India \\ ${ }^{1}$ sukantathomas@gmail.com, \\ ${ }^{2}$ sk7632@gmail.com, ${ }^{3}$ mailarindambiswas@yahoo.co.in, \\ parthabanerjee21@gmail.com, ${ }^{4}$ akbece12@yahoo.com,
}

\begin{abstract}
In this paper we propose a method to overcome the SNR-wall for the detection of OFDM signal by exploiting the autocorrelation property of the cyclic prefix in multipath fading channel. Required SNR for signal detection at a given number of observation samples for a fixed false alarm probability and detection probability has been found out. Our result shows that there is about $6 \mathrm{~dB}$ advantage for the autocorrelation based detector over conventional energy detector for noise uncertainty parameter $(U)$ equals to 3 with $10^{4}$ signal samples. Analytical results have been verified with simulation and close agreement has been found.
\end{abstract}

Keywords - Auto-correlation based detection, cyclic prefix, noise uncertainty, SNR-wall

\section{INTRODUCTION}

Spectrum sensing which is basically the detection of the presence or absence of a primary signal in a given frequency band, is one of the main functions of the Cognitive Radios (CR) [1], [2]. Many different kind of spectrum sensing techniques are already available in literature e.g. energy sensing, matched filter detection, cyclostationary detection etc. Among the different sensing techniques [3], [4] energy detector [1]performs optimally if both signal and noise are white Gaussian and most importantly the exact noise variance is known in advance. When the noise variance is perfectly known, the detector can detect the presence of a signal at any arbitrary low signal-to-noise ratio $(S N R)$ for given false alarm and detection probabilities by increasing the sensing time. But it is impossible to figure out the exact noise variance from a finite number of noise samples. R. Tandra and A. Sahai in their paper [2] have introduced a mathematical model for the uncertainty in noise process. It shows that the uncertainty in noise imposes a fundamental limitation on detector performance which cannot be overcome by increasing the sensing time. The nominal SNR threshold below which this phenomenon is observed is called the SNR-wall of the detector. In [5] it has been briefly mentioned that crosscorrelation among signals may be one of the way to overcome noise uncertainty problem. Some significant studies on noise uncertainties and SNR wall issues have been made in [3

Received: July 5, 2019

Reviewed: October 15, 2019

Accepted: October 29, 2019 
- 12]. R. Tandra and A. Sahai in [13] introduced the idea of macroscale features and showed that the features can be used to construct signals that can evade SNR-walls. This method is not very practical as the signal form the primary users need to be adaptive as mentioned in [14-16]. In [17] it is shown that the SNR-wall can be avoided if the variance of the noise power reduces when closely spaced observation samples are taken. In paper [18] the authors have presented a method to reduce the SNR-wall for energy detection by using the cross-correlation of the signals received by multiple receivers. But a single antenna receiver cannot be used to overcome the SNR-wall problem using this method. Instead of cross-correlation, the autocorrelation property can also be used for signals which exhibit some periodic property and this would not require multiple receivers. Several notable works have been done in recent past on relevant issues on spectrum sensing regarding noise uncertainty and SNR-wall problem. The paper [19] proposed to solve the SNR wall problem for energy detection, an improved energy detection (IED) algorithm based on non-linear stochastic resonance (SR) is proposed in this paper which describes a non-linear physical phenomenon whose properties is the SNR gain of certain non-linear systems. The work in [20] presents a fast sequency-ordered complex Hadamard transform (FSCHT)-based Parzen window entropy detection technique (PWED) for spectrum sensing which shown a substantial improvement in the SNR wall in the presence of noise uncertainty. The work in [21] proposed novel generalized energy detector (GED) for wide-band spectrum sensing which does not suffer from SNR wall problem and outperforms the existing signal detectors. Chaotic stochastic resonance (CSR) has been proposed in [22] where SNR wall in the traditional energy detector decreases by introducing the received signal into CSR system. The paper [23] studied the spectrum sensing in multiple transmit power levels scenario considering the noise uncertainty at the secondary user (SU) side. They not only proved that the energy detection is the optimal for both PU detection and power level recognition but also showed that SNR walls also exist for multiple transmit power levels scenario. It is also found that, there exist multiple SNR walls, two for each power level. The exact positions of those SNR walls are also computed. It is well known that cooperative energy detection algorithms can improve the detection probability significantly over conventional single energy detector. The paper [24] investigates the SNR wall phenomenon in a new perspective and analyzes it under typical cooperative energy detection algorithms such as AND/OR hard decision and equal gain combining soft decision. To keep a constant detection probability, three kinds of detection threshold viz. conservative, traditional and self-adaptive threshold, are studied and their influence are compared to find out the impact of SNR wall phenomenon. The work in [25] too extends the idea of SNR Wall phenomenon for energy detection in the multiple sensor scenarios by using a hybrid approach with auxiliary noise estimation under a typical flat fading channel condition. SNR Wall expression is derived for multi-sensor energy detection and proved to be independent of the number of sensors. The distribution of the uncertainty of the noise variance estimate is derived for auxiliary Gaussian noise samples. It is shown that the noise uncertainty can be reduced by increasing the number of samples used for noise variance estimation, but the number of samples/slot used for noise estimation exponentially increases as the SNR Wall condition becomes more stringent.

In this paper, we propose a method using the autocorrelation property of a cyclic prefix based OFDM signal to overcome the SNR-wall for signal detection. Autocorrelation based spectrum sensing for OFDM signal has been described in [26-28], but in their calculations the authors do not consider any uncertainty in the noise. This idea in [29] is already proposed, but detailed mathematical analysis has not been included there and also the results have not been validated by Monte Carlo simulation. For sufficiently large observation samples (>1000 samples) and at moderate cyclic prefix to OFDM symbol length ratio (>15\%) this method provides lower $S N R$ requirement in signal detection for given false alarm and detection probabilities at a fixed noise uncertainty. It is important 
that the OFDM symbol length and the cyclic prefix length must be known to the detector. It is assumed in our analysis that the channel fading is frequency selective and slowly varying with time.

The rest of the paper is organized as follows: Section IIA provides a brief description of energy detection performance in presence of noise uncertainty. In Section IIB we propose a scheme to overcome the SNR-wall using the autocorrelation property of an OFDM signal. Simulation results are given in Section III and conclusions are drawn in Section IV.

\section{ANALYTICAL MODEL AND STATISTICS}

\section{A. Fundamental Limit on Energy Detection}

When the noise variance is exactly known for an energy detector, the number of received samples and the $S N R$ of the signal must satisfy the relation [5]

$N=\frac{2\left(Q^{-1}\left(P_{D, d e s}\right)-Q^{-1}\left(P_{F A, d e s}\right)(1+S N R)\right)^{2}}{S N R^{2}}(1)$

at a desired false alarm probability $P_{F A \text {,des }}$ and a desired detection probability $P_{D, \text { des }}$. From (1) it is evident that $S N R \rightarrow 0$ as $N \longrightarrow \infty$. So the signal could be detected for arbitrarily low $S N R$ by increasing the sensing time. In presence of noise uncertainty and at a low $S N R$ the above equation will be modified to [5]:

$N=\frac{2\left(U Q^{-1}\left(P_{F A, d e s}\right)-Q^{-1}\left(P_{D, d e s}\right)\right)^{2}}{(S N R-(U-1))^{2}}$

where $U$ is the noise uncertainty parameter [5]. From (2) it is clear that $S N R \rightarrow(U-1)$ as $N \rightarrow \infty$. So the signal cannot be detected robustly for $S N R<(U-1)$ even by increasing the number of samples $N$. This lower limit of the SNR is called the SNR-wall.

B. Overcoming the SNR-Wall by Autocorrelation of Cyclic Prefix Based OFDM Signal

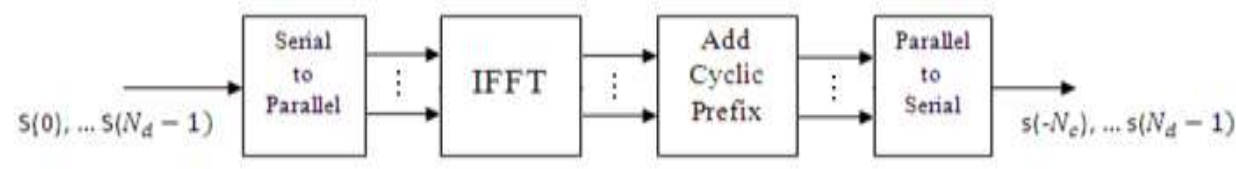

Fig. 1 Block diagram of an OFDM transmitter

The simplified block diagram of an OFDM transmitter is shown in Fig. 1. The block of $N_{d}$ symbol constellations $S(0), S(1), \ldots, S\left(N_{d}-1\right)$ are first converted into parallel symbols and then they are modulated by different subcarriers by passing those symbols through an IFFT function block. The IFFT yields the sequences $s(0), s(1), \ldots, s\left(N_{d}-1\right)$, where

$s(n)=\frac{1}{\sqrt{N_{d}}} \sum_{k=0}^{N_{d}-1} S(k) e^{j 2 \pi n k / N_{d}}, \quad 0 \leq n \leq N_{d}-1$

After IFFT, the signal sequence is appended by another sequence of $N_{c}$ samples, called the cyclic prefix, at the beginning of each block which consists of the last $N_{c}$ samples of the original signal sequence. This yields a new sequence of length $N_{s}=N_{d}+N_{c}$. This combined sequence is then converted back to serial stream of samples and transmitted. The block of samples: 


$$
\underbrace{S\left(-N_{c}\right), \ldots, S(-1)}_{\text {Cyclic Prefix }}, \underbrace{S(0), S(1), S(2) \ldots S\left(N_{d}-1\right)}_{\text {Data part }}
$$

at the output of the transmitter is called an OFDM symbol. Without the loss of generality we can change the coordinate points of the OFDM symbol and represent the transmitted samples by $x_{0}, x_{1}, \ldots, x_{N_{S}-1}$. Let the discrete-time channel impulse response is given by $\boldsymbol{h}=\left[h_{0}, h_{1}, \ldots h_{L-1}\right]$, where $L$ is the number of multipath component. Each $h_{i}$ 's are independent and Rayleigh distributed. The received signal at the secondary receiver is given by

$y_{i}=\sum_{k=0}^{L-1} h_{k} x_{i-k}+n_{i}, \quad i=0,1, \ldots, N_{s}-1(4)$

where $n_{i}$ is the additive white Gaussian noise with zero mean and variance $\sigma_{n}^{2}$. Because of multipath fading it is difficult to identify a particular OFDM symbol as the symbols travelling along different paths tend to overlap with each other. But there is still a correlation among the samples at a delay $N_{d}$ for some part of the signal because of the cyclic prefix property. We take any arbitrary $N$ number of consecutive samples from the received signal and compute the following metric:

$T=\frac{1}{N} \sum_{i=0}^{N-1} y_{i} y_{i+N_{d}}^{*}$

To calculate the distribution of $T$, let $z_{i}=y_{i} y_{i+N_{d}}^{*}$. Then,

$$
\operatorname{Re}(T)=\frac{1}{N} \sum_{i=0}^{N-1} \bar{z}_{i}
$$

We denote $\bar{z}$ as the real part of $z . \operatorname{Re}(T)$ is taken as the test statistic in this case. For large $N$ it can be shown by the central limit theorem that $\operatorname{Re}(T)$ follows Gaussian distribution with

$$
\begin{aligned}
& E[\operatorname{Re}(T)]=\frac{1}{N} \sum_{i=0}^{N-1} E\left(\bar{z}_{i}\right)=E\left(\bar{z}_{i}\right) \\
& \operatorname{Var}[\operatorname{Re}(T)]=\frac{1}{N} \operatorname{Var}\left(\bar{z}_{i}\right)(7 \mathrm{~b})
\end{aligned}
$$

It can be proved that (details shown in the appendix)

$$
E\left(\bar{z}_{i}\right)=\mu \sigma_{r}^{2}
$$

and, $\operatorname{Var}\left(\bar{z}_{i}\right) \approx \frac{\sigma_{r}^{4}}{2}+\sigma_{r}^{2} \sigma_{n}^{2}+\frac{\sigma_{n}^{4}}{2}(9)$

where $\mu=\frac{N_{c}}{N_{c}+N_{d}}$ and $\sigma_{r}^{2}$ is the variance of the received signal samples without the noise i.e. $\sigma_{r}^{2}=\operatorname{Var}\left(\sum_{k=0}^{L-1} h_{k} x_{i-k}\right)$

Hence, $\quad \operatorname{Re}(T) \mid H_{0} \sim \mathcal{N}\left(0, \frac{1}{2 N} \sigma_{n}^{4}\right)(10 \mathrm{a})$

and, $\quad \operatorname{Re}(T) \mid H_{1} \sim \mathcal{N}\left(\mu \sigma_{r}^{2}, \frac{1}{N}\left(\frac{\sigma_{r}^{4}}{2}+\sigma_{r}^{2} \sigma_{n}^{2}+\frac{\sigma_{n}^{4}}{2}\right)\right)(10 \mathrm{~b})$

We now select a proper threshold $\gamma$ for the desired false alarm probability $P_{F A, d e s}$. Then from (10a),

$$
P_{F A, \text { des }}=Q\left(\frac{\gamma}{\sqrt{\frac{1}{2 N}} \sigma_{n}^{2}}\right)(11)
$$

Setting the threshold at $\gamma$ the expression for the desired probability of detection $P_{D \text {,des }}$ can be written from (10b) as, 
$P_{D, \text { des }}=Q\left(\frac{\gamma-\mu \sigma_{r}^{2}}{\sqrt{\frac{1}{N}\left(\frac{\sigma_{r}^{4}}{2}+\sigma_{r}^{2} \sigma_{n}^{2}+\frac{\sigma_{n}^{4}}{2}\right)}}\right)(12)$

In the presence of noise uncertainty $U$, setting the desired detection probability at the worst case we can write

$P_{D, \text { des }}=Q\left(\frac{U \gamma-\mu \sigma_{r}^{2}}{\sqrt{\frac{1}{N}\left(\frac{\sigma_{r}^{4}}{2}+\sigma_{r}^{2} \sigma_{n}^{2}+\frac{\sigma_{n}^{4}}{2}\right)}}\right)$ (13)

From (11), $\gamma=\frac{1}{\sqrt{2 N}} \sigma_{n}^{2} B$, where $B=Q^{-1}\left(P_{F A, d e s}\right)$.

Now putting the value of $\gamma$ in (13) we obtain

$\frac{A^{2}}{N}\left(\frac{S N R^{2}}{2}+S N R+\frac{1}{2}\right)=\frac{U^{2} B^{2}}{2 N}+\mu^{2} S N R^{2}-\frac{2 \mu U B}{\sqrt{2 N}} \operatorname{SNR}(14)$

where $A=Q^{-1}\left(P_{D, \text { des }}\right)$ and $S N R=\frac{\sigma_{r}^{2}}{\sigma_{n}^{2}}$. At low $S N R$ the above equation can be approximated for $N>1000$ and at moderate $\mu(>0.15)$ as:

$\frac{A^{2}}{2 N}=\frac{U^{2} B^{2}}{2 N}+\mu^{2} S N R^{2}-\frac{2 \mu U B}{\sqrt{2 N}} S N R(15)$

We can derive from (15),

$N=\frac{\left(U Q^{-1}\left(P_{F A, d e s}\right)-Q^{-1}\left(P_{D, d e s}\right)\right)^{2}}{2 \mu^{2} \cdot S N R^{2}}(16)$

From (16) it is clear that $S N R \rightarrow 0$ as $N \rightarrow \infty$. So the signal could be detected robustly for any arbitrarily low $S N R$ by increasing the number of samples $N$ and this overcomes the SNR-wall.

\section{SIMULATIONS AND DISCUSSIONS}

In this section, we compare the analytical expression (16) for autocorrelation based detector with the conventional energy detector. Fig. 2 illustrates the graphs of required $S N R$ in $\mathrm{dB}$ versus the number of samples $N$ needed for detection for different sets of values of $U$ to reach a given set of false alarm and detection probabilities $\left(P_{F A, \text { des }}=\right.$ $0.01, P_{D, \text { des }}=0.99$ ) of an energy detector vide equation (2). It is evident from the curves corresponding to Fig. 2 that for $U=1$ any arbitrary pair $\left(P_{F A, d e s}, P_{D, d e s}\right)$ can always be reached by changing the sensing time (i.e. number of samples $N$ ) at all values of $S N R$ 


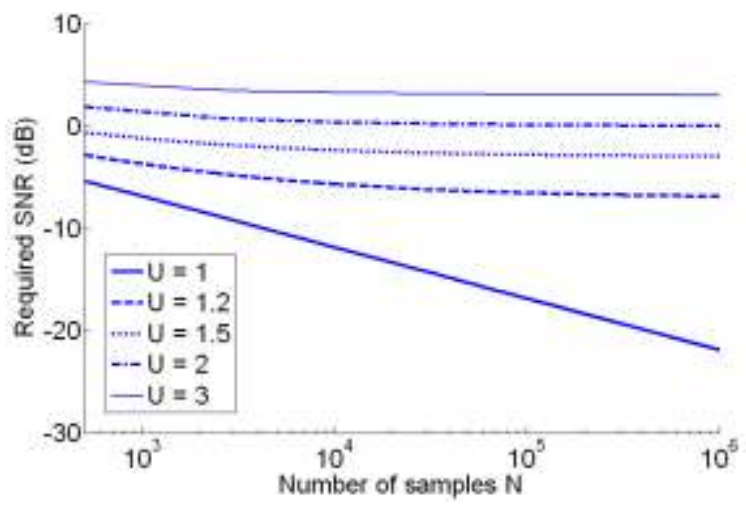

Fig. 2 Required $S N R$ in $\mathrm{dB}$ versus number of samples $N$ at $P_{F A, d e s}=0.01$ and $P_{D \text {,des }}=$ 0.99 for different values noise uncertainty $U$ in energy detection

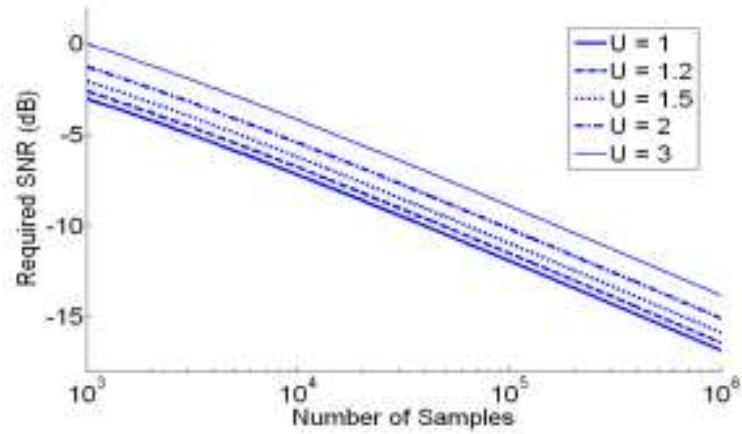

Fig. 3 Required $S N R$ in $\mathrm{dB}$ versus number of samples at $P_{F A \text {,des }}=0.01$ and $P_{D \text {,des }}=$ 0.99 for different values of noise uncertainty $U$ in our proposed method using the autocorrelation of OFDM signal $\left(N_{c}=80, N_{s}=512\right)$

but when $U>1$ the curves show asymptotic behavior converging toward a minimum limit. Beyond that limit of $S N R$, the signal cannot be detected reliably. In Fig. 3, the analytical results of our proposed method vide equation (16) have been plotted for OFDM signal in multipath fading channel. We select the OFDM parameters specifications $N_{S}=512$ and $N_{c}=80$. In this case even for large uncertainty parameter $(U>1)$, the required $S N R$ curves do not show asymptotic behavior. So for any pair $\left(P_{F A, d e s}, P_{D, d e s}\right)$, signal of arbitrary $S N R$ can be detected by increasing the sensing time.

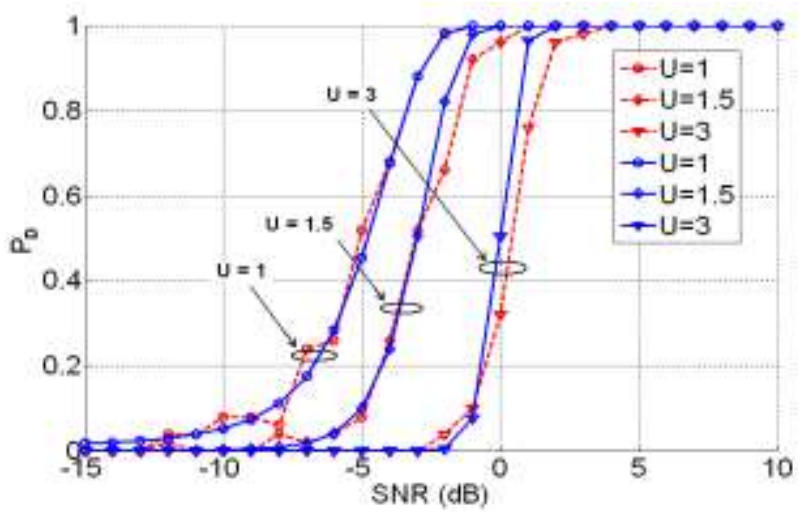

Fig. 4. $P_{D}$ versus $S N R$ obtained from simulation (red dotted curves) and theoretical analysis (blue continuous curves) for fixed $P_{F A}=0.01$ for different values of noise uncertainty values $U$ in our proposed method using the autocorrelation of OFDM signal with received signal samples $N=1000$. 
We have also carried out a simulation to detect the randomly generated OFDM signal by Monte Carlo method and verified the simulated results with our analytical results. Here the detection probability $P_{D}$ is plotted with respect to the $S N R$ of the signal keeping false alarm probability $P_{F A}$ constant at 0.01 for different uncertainty parameter $U$ with number of sensing samples $N=10^{3}$ for auto-correlation based OFDM detector. From Fig. 4 we see that the simulated values closely match with that of the analytically obtained values.

\section{CONCLUSIONS}

In this work, we show that the SNR-wall for signal detection can be avoided by exploiting the autocorrelation property of a cyclic prefix based OFDM signal in a fading channel. It is shown that with $10^{3}$ signal samples and a noise uncertainty parameter $U=3$, the autocorrelation based detector can provide about $6 \mathrm{~dB}$ advantage over conventional energy detector at a given false alarm probability of 0.01 and detection probability of 0.99 . Simulation results show good agreement with the analytical formula derived in this work

\section{APPENDIXES}

Derivation for the Expression of $E\left(\bar{z}_{i}\right)$ and $\operatorname{Var}\left(\bar{z}_{i}\right)$ :

Since $z_{i}=y_{i} y_{i+N_{d}}^{*}$ we can write

$$
\begin{gathered}
\bar{z}_{i}=\operatorname{Re}\left\{\left(\bar{y}_{i}+j \tilde{y}_{i}\right)\left(\bar{y}_{i+N_{d}}-j \tilde{y}_{i+N_{d}}\right)=\bar{y}_{i} \bar{y}_{i+N_{d}}+\tilde{y}_{i} \tilde{y}_{i+N_{d}}\right. \\
E\left(\bar{z}_{i}\right)=E\left(\bar{y}_{i} \bar{y}_{i+N_{d}}\right)+E\left(\tilde{y}_{i} \tilde{y}_{i+N_{d}}\right) \\
E\left(\bar{y}_{i} \bar{y}_{i+N_{d}}\right)=E\left[\left(\bar{r}_{i}+\bar{n}_{i}\right)\left(\bar{r}_{i+N_{d}}+\bar{n}_{i+N_{d}}\right)\right]=E\left(\bar{r}_{i} \bar{r}_{i+N_{d}}\right)
\end{gathered}
$$

Where $r_{i}=\sum_{k=0}^{L-1} h_{k} x_{i-k}$

$$
\begin{aligned}
& \text { Similarly, } E\left(\tilde{y}_{i} \tilde{y}_{i+N_{d}}\right)=E\left(\tilde{r}_{i} \tilde{r}_{i+N_{d}}\right) \\
& \begin{array}{r}
\text { Now } \bar{r}_{i}=\operatorname{Re}\left(\sum_{k=0}^{L-1} h_{k} x_{i-k}\right)=\sum_{k=0}^{L-1} \operatorname{Re}\left(h_{k} x_{i-k}\right) \\
=\sum_{k=0}^{L-1}\left(\bar{h}_{k} \bar{x}_{i-k}-\tilde{h}_{k} \tilde{x}_{i-k}\right) \\
\text { And } \bar{r}_{i+N_{d}}=\sum_{k=0}^{L-1}\left(\bar{h}_{k} \bar{x}_{i+N_{d}-k}-\tilde{h}_{k} \tilde{x}_{i+N_{d}-k}\right) \\
\qquad E\left(\bar{r}_{i} \bar{r}_{i+N_{d}}\right)=\sum_{k} \sum_{l}^{L} E\left[\left(\bar{h}_{k} \bar{x}_{i-k}-\tilde{h}_{k} \tilde{x}_{i-k}\right)\left(\bar{h}_{l} \bar{x}_{i+N_{d}-l}-\tilde{h}_{l} \tilde{x}_{i+N_{d}-l}\right)\right] \\
=\sum_{k} \sum_{l} E\left[\left(\bar{h}_{k} \bar{h}_{l} \bar{x}_{i-k} \bar{x}_{i+N_{d}-l}+\tilde{h}_{k} \tilde{h}_{l} \tilde{x}_{i-k} \tilde{x}_{i+N_{d}-l}-\tilde{h}_{k} \bar{h}_{l} \tilde{x}_{i-k} \bar{x}_{i+N_{d}-l}\right.\right. \\
\left.\left.\quad-\bar{h}_{k} \tilde{h}_{l} \bar{x}_{i-k} \tilde{x}_{i+N_{d}-l}\right)\right]
\end{array}
\end{aligned}
$$

For $k \neq l$ all the above four terms will be zero. The first two terms will be nonvanishing if $k=l$ and $x_{i-k} \in C P$ where CP denotes the set of samples of $x_{i}{ }^{\prime}$ s which belong to the cyclic prefix region.

$$
\begin{gathered}
E\left(\bar{r}_{i} \bar{r}_{i+N_{d}}\right)=\sum_{k} E\left(\bar{h}_{k}{ }^{2} \bar{x}_{i-k}{ }^{2}+\tilde{h}_{k}{ }^{2} \tilde{x}_{i-k}{ }^{2}\right) \times P\left(x_{i-k} \in C P\right) \\
=\sum_{k} E\left(\bar{h}_{k}{ }^{2} \bar{x}_{i-k}{ }^{2}+\tilde{h}_{k}{ }^{2} \tilde{x}_{i-k}{ }^{2}\right) \times \frac{N_{c}}{N_{c}+N_{d}} \\
=\mu \sum_{k}\left(\bar{h}_{k}{ }^{2} \frac{\sigma_{x}^{2}}{2}+\tilde{h}_{k}{ }^{2} \frac{\sigma_{x}^{2}}{2}\right)=\mu \sum_{k}\left(\bar{h}_{k}{ }^{2}+\tilde{h}_{k}{ }^{2}\right) \frac{\sigma_{x}^{2}}{2} \\
=\mu \sum_{k}\left|h_{k}\right|^{2} \frac{\sigma_{x}^{2}}{2} \text { where } \mu=\frac{N_{c}}{N_{c}+N_{d}}
\end{gathered}
$$


$=\mu \frac{\sigma_{r}^{2}}{2}$ where $\sigma_{r}^{2}=\sum_{k}\left|h_{k}\right|^{2} \sigma_{x}^{2}$ is the received signal power.

Similarly, $E\left(\tilde{r}_{i} \tilde{r}_{i+N_{d}}\right)=\mu \frac{\sigma_{r}^{2}}{2}$

$$
\begin{gathered}
E\left(\bar{z}_{i}\right)=E\left(\bar{r}_{i} \bar{r}_{i+N_{d}}\right)+E\left(\tilde{r}_{i} \tilde{r}_{i+N_{d}}\right)=\mu \frac{\sigma_{r}^{2}}{2}+\mu \frac{\sigma_{r}^{2}}{2}=\mu \sigma_{r}^{2} \\
\text { Now, } \operatorname{Var}\left(\bar{z}_{i}\right)=E\left(\bar{z}_{i}^{2}\right)-\left[E\left(\bar{z}_{i}\right)\right]^{2} \\
\quad E\left(\bar{z}_{i}^{2}\right)=E\left[\left(\bar{y}_{i} \bar{y}_{i+N_{d}}+\tilde{y}_{i} \tilde{y}_{i+N_{d}}\right)^{2}\right] \\
=E\left(\bar{y}_{i}{ }^{2} \bar{y}_{i+N_{d}}{ }^{2}\right)+E\left(\tilde{y}_{i}{ }^{2} \tilde{y}_{i+N_{d}}{ }^{2}\right)+2 E\left(\bar{y}_{i} \bar{y}_{i+N_{d}}\right) E\left(\tilde{y}_{i} \tilde{y}_{i+N_{d}}\right) \\
=E\left(\bar{y}_{i}{ }^{2} \bar{y}_{i+N_{d}}{ }^{2}\right)+E\left(\tilde{y}_{i}{ }^{2} \tilde{y}_{i+N_{d}}{ }^{2}\right)+\mu^{2} \frac{\sigma_{r}^{4}}{2}
\end{gathered}
$$

Now, $y_{i}=r_{i}+n_{i}$

$$
\begin{gathered}
\bar{y}_{i}=\bar{r}_{i}+\bar{n}_{i} \\
\bar{y}_{i}{ }^{2}=\bar{r}_{i}{ }^{2}+\bar{n}_{i}{ }^{2}+2 \bar{r}_{i} \bar{n}_{i} \\
E\left(\bar{y}_{i}{ }^{2} \bar{y}_{i+N_{d}}{ }^{2}\right)=E\left(\bar{r}_{i}{ }^{2} \bar{r}_{i+N_{d}}{ }^{2}+\bar{r}_{i}^{2} \bar{n}_{i+N_{d}}{ }^{2}+\bar{n}_{i+N_{d}}{ }^{2} \bar{r}_{i+N_{d}}{ }^{2}+\bar{n}_{i}{ }^{2} \bar{n}_{i+N_{d}}{ }^{2}\right) \\
E\left(\bar{r}_{i}{ }^{2} \bar{r}_{i+N_{d}}{ }^{2}\right)=E\left(\bar{r}_{i}{ }^{2}\right) E\left(\bar{r}_{i+N_{d}}{ }^{2}\right)+2 E\left(\bar{r}_{i} \bar{r}_{i+N_{d}}\right) E\left(\bar{r}_{i} \bar{r}_{i+N_{d}}\right)-2\left\{E\left(\bar{r}_{i}\right)\right\}^{2}\left\{E\left(\bar{r}_{i+N_{d}}\right)\right\}^{2} \\
E\left(\bar{r}_{i}\right)=E\left[\sum_{k=0}^{L-1}\left(\bar{h}_{k} \bar{x}_{i-k}-\tilde{h}_{k} \tilde{x}_{i-k}\right)\right] \\
=\sum_{i=0}^{L-1}\left[\bar{h}_{k} E\left(\bar{x}_{i-k}\right)-\tilde{h}_{k} E\left(\tilde{x}_{i-k}\right)\right]=0 \text { (i.i.d Gaussian) }
\end{gathered}
$$

Similarly, $E\left(\bar{r}_{i+N_{d}}\right)=0$

$$
\begin{gathered}
E\left(\bar{r}_{i}^{2}\right)=E\left[\left\{\sum_{k=0}^{L-1}\left(\bar{h}_{k} \bar{x}_{i-k}-\tilde{h}_{k} \tilde{x}_{i-k}\right)\right\}^{2}\right] \\
=E\left[\left\{\sum_{k} \sum_{l}\left(\bar{h}_{k} \bar{x}_{i-k}-\tilde{h}_{k} \tilde{x}_{i-k}\right)\left(\bar{h}_{l} \bar{x}_{i-l}-\tilde{h}_{l} \tilde{x}_{i-l}\right)\right\}\right]
\end{gathered}
$$

The above term will be non-vanishing if $l=k$ or $l=k-N_{d}$ and $x_{i-k} \in C P$. Therefore

$$
\begin{aligned}
E\left(\bar{r}_{i}^{2}\right)=E\left[\sum_{k=0}^{L-1}\left(\bar{h}_{k} \bar{x}_{i-k}-\tilde{h}_{k} \tilde{x}_{i-k}\right)^{2}\right] \\
\quad+E\left[\sum_{k=0}^{L-1}\left(\bar{h}_{k} \bar{x}_{i-k}-\tilde{h}_{k} \tilde{x}_{i-k}\right)\left(\bar{h}_{k-N_{d}} \bar{x}_{i+N_{d}-k}-\tilde{h}_{k-N_{d}} \tilde{x}_{i+N_{d}-k}\right)\right] \\
\quad \times P\left(x_{i-k} \in C P\right)
\end{aligned}
$$

The second term above in the R.H.S is zero since $\bar{h}_{k-N_{d}}=\widetilde{h}_{k-N_{d}}=0$ for $k=0,1, \ldots L$ assuming $L<N_{d}$.

$$
\begin{gathered}
E\left(\bar{r}_{i}{ }^{2}\right)=E\left[\sum_{k=0}^{L-1}\left(\bar{h}_{k} \bar{x}_{i-k}-\tilde{h}_{k} \tilde{x}_{i-k}{ }^{2}\right]\right. \\
=\sum_{k} E\left(\bar{h}_{k}{ }^{2} \bar{x}_{i-k}{ }^{2}+\tilde{h}_{k}{ }^{2} \tilde{x}_{i-k}{ }^{2}-2 \bar{h}_{k} \tilde{h}_{k} \bar{x}_{k-i} \tilde{x}_{k-i}\right) \\
=\sum_{k}\left(\bar{h}_{k}{ }^{2}+\tilde{h}_{k}{ }^{2}\right) \frac{\sigma_{x}^{2}}{2}=\sum_{k}\left|h_{k}\right|^{2} \frac{\sigma_{x}^{2}}{2}=\frac{\sigma_{r}^{2}}{2}
\end{gathered}
$$

Similarly, $E\left(\bar{r}_{i+N_{d}}^{2}\right)=\frac{\sigma_{r}^{2}}{2}$

$$
E\left(\bar{r}_{i}^{2} \bar{r}_{i+N_{d}}^{2}\right)=\frac{\sigma_{r}^{2}}{2} \cdot \frac{\sigma_{r}^{2}}{2}+2 \frac{\mu \sigma_{r}^{2}}{2} \cdot \frac{\mu \sigma_{r}^{2}}{2}=\frac{\sigma_{r}^{4}}{4}+\frac{\mu^{2} \sigma_{r}^{4}}{2}
$$




$$
\begin{aligned}
& \qquad E\left(\bar{r}_{i}^{2} \bar{n}_{i+N_{d}}{ }^{2}\right)= \\
& E\left({\overline{r_{i}}}^{2}\right) E\left(\bar{n}_{i+N_{d}}{ }^{2}\right)+2 E\left(\bar{r}_{i} \bar{n}_{i+N_{d}}\right) E\left(\bar{r}_{i} \bar{n}_{i+N_{d}}\right)-2\left\{E\left(\bar{r}_{i}\right)\right\}^{2}\left\{E\left(\bar{r}_{i+N_{d}}\right)\right\}^{2}=\frac{\sigma_{r}^{2}}{2} \cdot \frac{\sigma_{n}^{2}}{2} \\
& =\frac{\sigma_{r}^{2} \sigma_{n}^{2}}{4} \\
& \text { Similarly,E }\left(\bar{n}_{i}^{2} \bar{r}_{i+N_{d}}^{2}\right)=\frac{\sigma_{r}^{2} \sigma_{n}^{2}}{4} \text { and } E\left(\bar{n}_{i}^{2} \bar{n}_{i+N_{d}}{ }^{2}\right) \\
& =\frac{\sigma_{n}^{2}}{2} \cdot \frac{\sigma_{n}^{2}}{2}=\frac{\sigma_{n}^{4}}{4} . \text { Hence we can write } \\
& E\left(\bar{y}_{i}^{2} \bar{y}_{i+N_{d}}^{2}\right)=\frac{1}{4}\left\{\left(1+2 \mu^{2}\right) \sigma_{r}^{4}+2{\sigma_{r}^{2}}^{2} \sigma_{n}^{2}+\sigma_{n}^{4}\right\}(20)
\end{aligned}
$$

Similarly it can be shown:

$$
E\left(\tilde{y}_{i}^{2} \tilde{y}_{i+N_{d}}{ }^{2}\right)=\frac{1}{4}\left\{\left(1+2 \mu^{2}\right) \sigma_{r}^{4}+2 \sigma_{r}^{2} \sigma_{n}^{2}+\sigma_{n}^{4}\right\}(21)
$$

So from (19), (20) and (21),

$$
E\left(\bar{z}_{i}^{2}\right)=\frac{1}{2}\left\{\left(1+2 \mu^{2}\right) \sigma_{r}^{4}+2 \sigma_{r}^{2} \sigma_{n}^{2}+\sigma_{n}^{4}\right\}+\mu^{2} \frac{\sigma_{r}^{4}}{2}(22)
$$

And from (17), (18) and (22),

$$
\begin{gathered}
\operatorname{Var}\left(\bar{z}_{i}\right)=\frac{1}{2}\left\{\left(1+2 \mu^{2}\right) \sigma_{r}^{4}+2 \sigma_{r}^{2} \sigma_{n}^{2}+\sigma_{n}^{4}\right\}+\mu^{2} \frac{\sigma_{r}^{4}}{2}-\mu^{2} \sigma_{r}^{4} \\
=\frac{\left(1+\mu^{2}\right)}{2} \sigma_{r}^{4}+\sigma_{r}^{2} \sigma_{n}^{2}+\frac{\sigma_{n}^{4}}{2} \approx \frac{\sigma_{r}^{4}}{2}+\sigma_{r}^{2} \sigma_{n}^{2}+\frac{\sigma_{n}^{4}}{2}\left(\mu^{2} \ll 1\right)
\end{gathered}
$$

\section{REFERENCES}

[1] J. Mitola et al.,"Cognitive radio: Making software radios more personal,” IEEE pers Commun., vol. 6, no. 4, pp. 13-18, Aug. 1999.

[2] S. Haykin, "Cognitive radio: Brain-empowered wireless communications," IEEE Journal on Selected Areas in Communications, vol. 25, pp. 201-220, February 2005.

[3] B. Wang and K. J. R. Liu, "Advances in cognitive radio networks: A survey," IEEE J. Sel. Topics Signal Process. , vol. 5, no. 1, pp. 5-23, vol. 5, no. 1, pp. 5-23, Jan. 2011.

[4] T Yucek and H. Arslan," A survey of spectrum sensing algorithms for cognitive radio applications," IEEE Communications Surveys \& Tutorials, vol. 11,no. 1,pp. 116-130, 2009.

[5] H. Urkowitz, "Energy detection of unknown deterministic signals," Proc. IEEE, vol. 55, no. 4, pp. 523$531,1967$.

[6] Naveen Chandra Arya et al. ," A Review on Cooperative Communication in Cognitive Radio Network Using Relay," IJCTSNS, Vol. 5, No. 2, pp. 15-20, Sep 2017.

[7] Gaurav Singh Negi et al.," A Review: Routing Protocols for cognitive Radio Networks" IJWMCIS, Vol. 5, No. 1, pp. 1-6, April 2018.

[8] Uma Sharma, Poonam Mittal, Chander Kumar Nagpal, "A Novel Technique for Channel Allocation in Cognitive Radio Networks using Game Theory," IJWMCIS, Vol. 3, No. 2, pp. 47-54, Oct 2016.

[9] Ashish Bagwari, Jyotshana Kanti and Geetam Singh Tomar," New Cooperative Spectrum Detection Technique in Cognitive Radio Networks," IJWMCIS, Vol. 3, No. 1, pp. 43-58, April 2016.

[10] Uma Sharma, Poonam Mittal, Chander Kumar Nagpal," A Novel Technique for Channel Allocation in Cognitive Radio Networks using Game Theory,” IJWMCIS, Vol. 3, No. 2, pp. 47-54, Oct 2016.

[11] Hwi Jin Jang, Min Wook Kang, and Yun Won Chung, "An Opportunistic Routing Protocol based on Contact Ratio," IJPDCSD, Vol. 3, No. 1, pp. 21-26, December 2016.

[12] Y.P. Kumar," Identification of Malicious Packet Dropping in Ad-Hoc Networks," IJWMCIS, Vol. 3, No. 1, pp. 13-20, April 2016.

[13] R. Tandra and A. Sahai, "Overcoming SNR walls through macroscale features," in Proc.46th Annual Allerton Conf. Communication, Control, and Computing,2008, pp. 583-590.

[14] M. Alink, A. Kokkeler, G. Smit and B. Nauta, "Lowering the SNR wall for energy detection using cross-correlation," IEEE Trans. Vehicular Tech, Oct. 2011.

[15] Sengphil Hong ," Enhanced Re-route Filtering Scheme for Data Reporting in Wireless Sensor Networks" IJWMCIS, Vol. 3, No. 2, pp. 1-8, Oct 2016.

[16] Sandeep Kumar Agrawal and Ravi yadav ," Optimization in Cooperative Spectrum Sensing Techniques of CRN," IJWMCIS, Vol. 4, No. 1, pp. 9-18, April 2017.

[17] A. Mariani, A. Giorgetti, and M. Chiani, "SNR wall for energy detection with noise power estimation," in Proc. IEEE International Conference on Communications (ICC'11), pp. 1-6, June 2011.

[18] M. Alink, A. Kokkeler, G. Smit and B. Nauta, "Lowering the SNR wall for energy detection using cross-correlation,” IEEE Trans. Vehicular Tech, Oct. 2011. 
[19] Liu, Jin, and Zan Li. "Lowering the signal-to-noise ratio wall for energy detection using parameterinduced stochastic resonator." IET Communications 9.1 (2015): 101-107.

[20] Namburu, Swetha, et al. "Fast Sequency-Ordered Complex Hadamard Transform based Parzen Window Entropy detection for Spectrum Sensing in Cognitive Radio Networks."

[21] Bogale, Tadilo Endeshaw, Luc Vandendorpe, and Long Bao Le. "Wide-band sensing and optimization for cognitive radio networks with noise variance uncertainty." IEEE Transactions on Communications 63.4 (2015): 1091-1105.

[22] Di He, "Breaking the SNR wall of spectrum sensing in cognitive radio by using the chaotic stochastic resonance," Proceedings of IEEE International Symposium on Circuits and Systems (ISCAS)., 2010.

[23] Qian, C., Qian, H. and Gao, F., 2015, November. Spectrum sensing and SNR walls when primary user has multiple power levels. In Communications in China (ICCC), 2015 IEEE/CIC International Conference on (pp. 1-6). IEEE.

[24] Zeng, J. and Su, X., 2015, August. On SNR wall phenomenon under cooperative energy detection in spectrum sensing. In Communications and Networking in China (ChinaCom), 2015 10th International Conference on (pp. 53-60). IEEE.

[25] Dhakal, P., Rivello, D. and Garello, R., 2014, August. SNR Wall analysis of multi-sensor energy detection with noise variance estimation. In Wireless Communications Systems (ISWCS), 2014 11th International Symposium on (pp. 680-684). IEEE.

[26] S. Chaudhari, V. Koivunen, H. V. Poor, "Autocorrelation-Based Decentralized Sequential Detection of OFDM Signals in Cognitive Radios," IEEE Transactions on Signal Processing, July 2009.

[27] E. Axell and E. G. Larsson, "Optimal and near-optimal spectrum sensing of OFDM signals in AWGN channels," Workshop on Cognitive Information Processing (CIP), 2010.

[28] Pratima Manhasa, M.K Sonib," OFDM Performance Evaluation under Different Fading Channels AWGN,Rayleigh and Rician)," IJWMCIS, Vol. 5, No. 2, pp.19-24, October 2018.

[29] S. Karar, A. D. Barman, "Overcoming the SNR-wall for energy detection using the autocorrelation of cyclic prefix based OFDM signal," in Proc. IEEE National Conference on Communications (NCC2013), 2013. 